\title{
Anterolateral Portal Is Less Painful than Superolateral Portal in Knee Intra-Articular Injection
}

\author{
Sung Yup Lee, $\mathrm{MD}^{1}$, Kiran Kumar GN, MS (Ortho) ${ }^{1}$, Byung June Chung, $\mathrm{MD}^{2}$, Sang Wook Lee, $\mathrm{MD}^{1}$, and \\ Tae Kyun Kim, $\mathrm{MD}^{1}$ \\ ${ }^{1}$ Department of Orthopaedic Surgery, Seoul National University Bundang Hospital, Seongnam; ${ }^{2}$ Joint Reconstruction Center, Knee and Spine Hospital, Seoul, Korea
}

\begin{abstract}
Purpose: Intra-articular knee injections are commonly performed in clinical practice for treating various knee joint disorders such as osteoarthritis and rheumatoid arthritis. When selecting the portal for injection, not only intra-articular needle accuracy but also procedural pain should be taken into consideration. The purpose of this study was to determine whether injection through anterolateral portal provokes less pain and provides better pain relief compared to superolateral portal.

Materials and Methods: A total of 60 patients with primary osteoarthritis of the knee receiving intra-articular injections were randomized into 2 groups according to the type of portal approach; anterolateral or superolateral. All patients received hyaluronic acid ( $20 \mathrm{mg})$ and triamcinolone (40 $\mathrm{mg}$ ) as the first injection followed by second and third injections of hyaluronic acid on a weekly basis. Underlying knee pain, procedural pain, and knee pain at 4 weeks were evaluated using visual analogue scale (VAS).

Results: Injection through anterolateral portal provoked less pain (VAS, 1.5 \pm 1.3 ) than the superolateral portal (VAS, 1.5 vs. 2.7; p=0.004). No differences were found in the degree of pain relief at weeks between the two groups $(p=0.517)$.

Conclusions: We recommend the use of anterolateral portal for intra-articular knee injection as it provokes less pain and comparably short-term pain relief than the superolateral portal.
\end{abstract}

Keywords: Knee, Osteoarthritis, Intra-articular injection

\section{Introduction}

Intra-articular knee injection is a common, relatively simple and safe procedure done in an outpatient setting for various knee conditions. Injections of corticosteroids and hyaluronic acid (HA) are most common for the treatment of knee osteoarthritis (OA). Although several portals are available for knee injection ${ }^{1,2)}$, each has its own advantages and disadvantages. Accuracy of intra-

Received January 13, 2015; Accepted April 13, 2015

Correspondence to: Tae Kyun Kim, MD

Department of Orthopaedic Surgery, Seoul National University Bundang Hospital, 82 Gumi-ro 173beon-gil, Bundang-gu, Seongnam 13620,

Korea

Tel: +82-31-787-7196, Fax: +82-31-787-4056

E-mail: osktk@snubh.org

This is an Open Access article distributed under the terms of the Creative Commons Attribution Non-Commercial License (http://creativecommons.org/licenses/by-nc/4.0/) which permits unrestricted non-commercial use, distribution, and reproduction in any medium, provided the original work is properly cited. articular needle placement, portal site pain and experience of the clinician are important factors to be considered before selecting a portal for knee injection.

The two routinely used approaches for intra-articular knee injections are superolateral and anterolateral. The superolateral approach with the leg in extension is the most commonly studied approach in the literature ${ }^{3)}$. The anterolateral approach is familiar among knee surgeons due to its routine use in arthroscopic surgery. It allows the patient to remain in a sitting position with the knee bent, and bilateral injections can be performed with ease without changing the patient's position. It is, therefore, useful in patients whose knee cannot be extended, and furthermore, it does not require manipulation of the patella ${ }^{4)}$. It is reported that these arthroscopic approaches involve little pain or discomfort ${ }^{5}$. Accordingly, most of the previous studies stressed upon the accuracy of intra-articular needle placement. Few studies have described procedural pain and the degree of pain relief following intra-articular knee injection through various portals.

Hence, we sought to determine whether injection through the 
anterolateral portal provokes less pain and provides better pain relief than the superolateral portal.

\section{Materials and Methods}

This prospective randomized controlled study was undertaken in 60 patients from 30 June 2014 to 30 October 2014 at our tertiary care center. Patients were adequately educated regarding the nature of the study before the procedures. Written informed consent and clearance from the local ethical committee were obtained before the initiation of the study.

Inclusion criteria were patients with radiological KellgrenLawrence grade II or III OA knee and ability to give informed consent. Exclusion criteria were as follows: conditions other than primary OA, systemic diseases that may affect the results, HA and steroid injections within recent three months, allergy to HA injection, the use of warfarin or antiplatelet therapy, or the presence of any infection.

Patients were recruited in our outpatient department and randomly assigned to either the superolateral injection group or the anterolateral injection portal group using computer-generated permuted block randomization. There were 29 patients in the superolateral group and 31 patients in the anterolateral group.

Table 1. Comparisons of Demographic Features and Preoperative Status between the Anterolateral Portal Group and the Superolateral Portal Group

\begin{tabular}{lccc}
\hline \multicolumn{1}{c}{ Variable } & $\begin{array}{c}\text { Anterolateral } \\
\text { group } \\
(\mathrm{n}=31)\end{array}$ & $\begin{array}{c}\text { Superolateral } \\
\text { group } \\
(\mathrm{n}=29)\end{array}$ & p-value \\
\hline $\begin{array}{l}\text { Age (yr), mean (SD) } \\
\text { Sex (female) }\end{array}$ & $66.3(7.8)$ & $67.3(7.5)$ & 0.594 \\
OA severity by K-L grade & $28(90.3)$ & $28(93.3)$ & 0.613 \\
Patellofemoral joint ${ }^{\text {a) }}$ & & & \\
$\quad$ Grade I & $16(51.6)$ & $12(41.4)$ & 0.256 \\
$\quad$ Grade II & $12(38.7)$ & $11(37.9)$ & \\
$\quad$ Grade III & $3(9.7)$ & $6(20.7)$ & \\
Tibiofemoral joint & & & 0.603 \\
$\quad$ Grade II & $12(38.7)$ & $14(48.3)$ & \\
$\quad$ Grade III & $19(61.3)$ & $15(51.7)$ & \\
Pre-procedure pain (VAS), & $5.2(1.1)$ & $5.3(1.0)$ & 0.510 \\
mean (SD) & & & \\
\hline
\end{tabular}

Values are presented as number of patients (\%)

SD: standard deviation, OA: osteoarthritis, K-L: Kellgren-Lawrence, VAS: visual analog scale.

a) There were no patients with K-L grade IV for the patellofemoral joint and no patients with K-L grade I or IV for the tibiofibular joint.
Demographic features and preoperative status of the patients including the OA severity in the patellofemoral and tibiofemoral joints and mechanical tibiofemoral angle were compared between the two groups (Table 1). Underlying knee pain was recorded using visual analogue scale (VAS), where $0 \mathrm{~cm}=$ no pain and 10 $\mathrm{cm}=$ unbearable pain $^{6,7)}$.

All patients received weekly injections for three weeks in accordance with the assigned route on an outpatient basis under strict aseptic precautions. All procedures were carried out using 23 gauge needles blindly based on anatomic landmarks by an experienced surgeon. Injection through the anterolateral portal was given with the patient in a sitting position with the knee flexed to $90^{\circ}$ at $1 \mathrm{~cm}$ proximal to the joint line, lateral to the patellar tendon, the needle was directed towards the intercondylar notch (Fig. 1). Injection through the superolateral portal was performed with the patient in supine position. With the knee extended, the needle was inserted $1 \mathrm{~cm}$ above and $1 \mathrm{~cm}$ lateral to the superolateral margin of patella at a $45^{\circ}$ angle in the cephalolateral to caudomedial direction (Fig. 2). The first injection included HA $(20 \mathrm{mg})$ and triamcinolone $(40 \mathrm{mg}$ ) and subsequently, the second and third injections included only HA $(20 \mathrm{mg})$.

The primary outcome variable was the degree of pain measured at the portal site during the first injection, and the secondary outcome variable was the degree of pain relief, which was evaluated at 4 weeks after the last injection. Procedural pain and knee pain were evaluated using a $0-10$ VAS, where 0 indicates no pain and 10 indicates the most severe pain. In order to ensure the validity and reliability of pain evaluation, a single investigator (Lee SY) assessed pain levels for all patients. The investigator had substan-

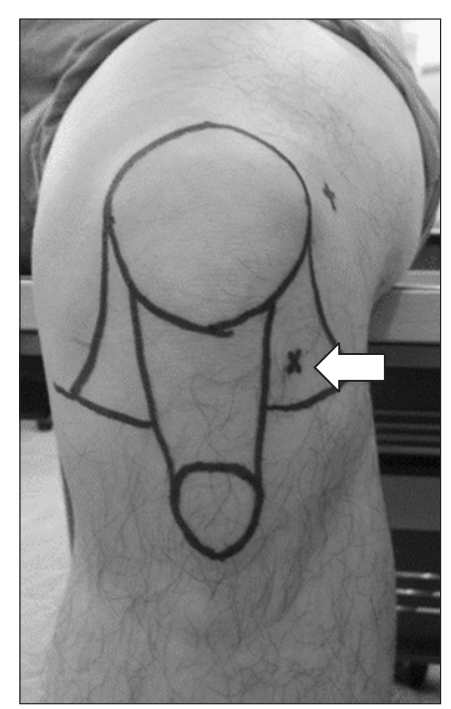

Fig. 1. Photograph showing the anterolateral portal site (block arrow) in the left knee with the patient sitting on the edge of a table. 


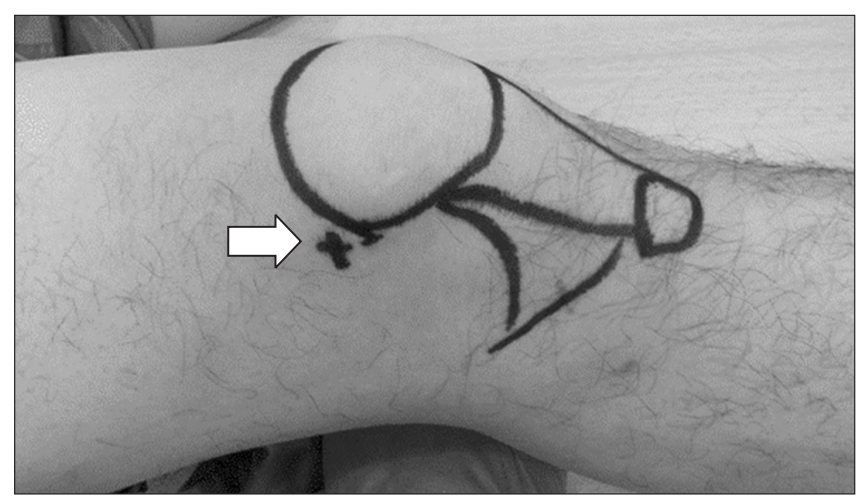

Fig. 2. Photograph showing the superolateral portal site (block arrow) in the right knee with the patient in the supine position.

tial experience in pain evaluation using a $0-10$ VAS. Any complications pertaining to the injection site were promptly noted.

Statistical analysis was done using the SPSS ver. 20.0 (IBM Co., Armonk, NY, USA) and p-values of $<0.05$ were considered statistically significant. The Chi-square test was used to compare categorical variables, and the Student $t$-test or paired $t$-test was used to compare numerical variables.

\section{Results}

Injection through the anterolateral portal provoked less pain than the superolateral portal, and no differences in the degree of pain relief at 4 weeks after last injection were found between the two groups. The mean procedural pain was lower in the anterolateral group than in the superolateral group (1.5 vs. $2.7, \mathrm{p}=0.004)$ (Table 2). No intergroup differences were found in pain level (2.9 vs. $3.1, \mathrm{p}=0.517)$ or the degree of pain relief ( 2.3 vs. $2.2, \mathrm{p}=0.883$ ) at 4 weeks after last injection. There were no immediate complications noted following injection, such as transient flushing reaction or erythema at the injection site.

\section{Discussion}

Intra-articular injection of the knee joint is commonly performed in clinical practice and is the most common invasive procedure in sports medicine ${ }^{4,8-11)}$. Although the accuracy of the intra-articular needle placement through various routes has been vastly studied, pain-related factors such as procedural pain were barely touched in the literature. The present study hypothesized that knee injection through the anterolateral portal would be less painful and provide better short-term pain relief than the superolateral portal. We found that injection through the anterolateral portal provoked less pain than the superolateral portal, and no
Table 2. Comparisons of Portal Site Pain and Pain Relief at Four Weeks after Injection between the Anterolateral Portal Group and the Superolateral Portal Group

\begin{tabular}{lccc}
\hline \multicolumn{1}{c}{ Variable $^{\text {a) }}$} & $\begin{array}{c}\text { Anterolateral } \\
\text { portal }(\mathrm{n}=31)\end{array}$ & $\begin{array}{c}\text { Superolateral } \\
\text { portal (n=29) }\end{array}$ & p-value \\
\hline Procedural pain & $1.5(1.3)$ & $2.7(1.5)$ & 0.004 \\
Pain at 4 wk & $2.9(1.5)$ & $3.1(1.2)$ & 0.517 \\
Reduction in pain score & $2.3(1.2)$ & $2.2(1.3)$ & 0.883 \\
at 4 wk from baseline & & & \\
\hline
\end{tabular}

Values are presented mean (standard deviation).

${ }^{\text {a) }}$ Visual analog scale.

differences in the degree of pain relief at 4 weeks after last injection were found between the two groups.

Findings in this study supported our primary hypothesis that injection through the anterolateral portal would provoke less pain as compared to the superolateral portal. Our findings are in contrast with a previous study reporting no significant difference in procedural pain between the modified anterolateral and lateral mid parapatellar portals ${ }^{12)}$. On the contrary, our findings are in line with another previous study on the anterior approach for knee arthrography, where significant reduction was observed in absolute and relative degree of pain for the anterolateral route compared with the anterior paramedian route ${ }^{5}$. We speculate that thinner soft tissue for the needle to transverse may be related to less pain in injection through the anterolateral portal, particularly when the knee is flexed to $90^{\circ}$. In addition, pain detected during the superolateral approach can be explained by accidental needle collision with the bone, quadriceps tendon, and suprapatellar synovium ${ }^{13)}$. Nonetheless, our study does not contain any data explaining why and how injections through the anterolateral portal provoke less pain than through the superolateral portal. Future studies are warranted to scrutinize this issue.

In our study, the degree of pain relief at 4 weeks of follow-up was comparable between the two groups. This does not support our secondary hypothesis that injections through the anterolateral portal offer better pain relief than through the superolateral portal. There are only two studies that compared clinical outcomes between different knee injection sites, and both studies found no significant difference between each other: lateral mid patellar injection vs. anterolateral injection ${ }^{12)}$ and infrapatellar injection vs. medial knee injection ${ }^{14)}$. On the other hand, several studies compared clinical outcomes of ultrasound-guided versus blinded injections and reported that ultrasound-guided injections provided better short-term clinical outcomes than blinded intraarticular knee injections ${ }^{15,16)}$. However, as the ultrasound-guided injection technique requires expensive devices and trained skills, 
its clinical and practical values should be evaluated according to the situation of each physician.

There are several limitations to our study. First, the accuracy of intra-articular needle placement was not confirmed. Before the initiation of this study, we considered ultrasonographic or radiographic confirmation of the accuracy of intra-articular needle placement, but it deemed impractical or unethical to expose study participants to additional expense or radiation hazards. Furthermore, we noted that even though the use of needle guidance might improve the accuracy of knee injections, insufficient evidence existed to prove that increased accuracy of knee injections would lead to improved therapeutic outcome. A previous study reported that blinded knee injections were reasonably accurate in the lateral injection sites ${ }^{5}$. Nonetheless, the lack of accuracy information should be noted to interpret our findings. A recent systematic review found that overall one in five blinded knee injections were inaccurate ${ }^{17)}$. In the systematic review, pooling data across studies suggested blinded knee injection at the superolateral portal site was most accurate (87\%) while injections through medial mid-patellar portal (64\%) and anterolateral portal $(70 \%)$ were less accurate. Therefore, whether injection through the anterolateral portal is more accurate than injection through the superolateral portal should be elucidated in future studies. Second, all the procedures were performed by a single experienced knee surgeon. The results of this study may have been affected by the experience of the surgeon, which may limit generalization of our findings. Third, only two lateral approaches were investigated in this study. Hence, studies evaluating the degree of pain through portals of different approaches are required. Finally, this study should be regarded as a preliminary study using a small sample size, which prompts future studies with larger sample sizes and sophisticated evaluation tools regarding needle placement accuracy. Because of the small sample size, we could not perform subgroup analyses according to various factors that could influence the technical difficulty during injection or the degree of pain relief such as body mass index, OA, severity, and the presence or severity of patellofemoral joint. Therefore, we could neither mention the effects of the confounders nor recommend individualized portal selection.

\section{Conclusions}

We recommend the anterolateral portal for intra-articular knee injections. It provokes less pain and provides better short-term pain relief than the superolateral portal. Randomized trials to evaluate pain upon multiple routes of injections as well as accu- racy of needle placement are needed.

\section{Conflict of Interest}

No potential conflict of interest relevant to this article was reported.

\section{References}

1. Courtney P, Doherty M. Joint aspiration and injection and synovial fluid analysis. Best Pract Res Clin Rheumatol. 2009; 23:161-92.

2. Lockman LE. Practice tips. Knee joint injections and aspirations: the triangle technique. Can Fam Physician. 2006; 52:1403-4.

3. Hermans J, Bierma-Zeinstra SM, Bos PK, Verhaar JA, Reijman M. The most accurate approach for intra-articular needle placement in the knee joint: a systematic review. Semin Arthritis Rheum. 2011;41:106-15.

4. Jackson DW, Evans NA, Thomas BM. Accuracy of needle placement into the intra-articular space of the knee. J Bone Joint Surg Am. 2002;84:1522-7.

5. Moser T, Moussaoui A, Dupuis M, Douzal V, Dosch JC. Anterior approach for knee arthrography: tolerance evaluation and comparison of two routes. Radiology. 2008;246:193-7.

6. Myles PS, Troedel S, Boquest M, Reeves M. The pain visual analog scale: is it linear or nonlinear? Anesth Analg. 1999; 89:1517-20.

7. Katz J, Melzack R. Measurement of pain. Surg Clin North Am. 1999;79:231-52.

8. Altman RD, Moskowitz R. Intraarticular sodium hyaluronate (Hyalgan) in the treatment of patients with osteoarthritis of the knee: a randomized clinical trial: Hyalgan Study Group. J Rheumatol. 1998;25:2203-12.

9. Leopold SS, Redd BB, Warme WJ, Wehrle PA, Pettis PD, Shott S. Corticosteroid compared with hyaluronic acid injections for the treatment of osteoarthritis of the knee: a prospective, randomized trial. J Bone Joint Surg Am. 2003; 85:1197-203.

10. Neustadt DH. Intra-articular injections for osteoarthritis of the knee. Cleve Clin J Med. 2006;73:897-8, 901-4, 906-11.

11. Schumacher HR, Chen LX. Injectable corticosteroids in treatment of arthritis of the knee. Am J Med. 2005;118:1208-14.

12. Chavez-Chiang CE, Sibbitt WL Jr, Band PA, Chavez-Chiang NR, Delea SL, Bankhurst AD. The highly accurate anteriolateral portal for injecting the knee. Sports Med Arthrosc 
Rehabil Ther Technol. 2011;3:6.

13. Sanchis-Alfonso V, Rosello-Sastre E, Subias-Lopez A. Neuroanatomic basis for pain in patellar tendinosis (“jumper's knee"): a neuroimmunohistochemical study. Am J Knee Surg. 2001;14:174-7.

14. Shah KD, Wright V. Intra-articular hydrocortisone in osteoarthrosis. Ann Rheum Dis. 1967;26:316-8.

15. Sibbitt WL Jr, Kettwich LG, Band PA, Chavez-Chiang NR, DeLea SL, Haseler LJ, Bankhurst AD. Does ultrasound guidance improve the outcomes of arthrocentesis and corticoste- roid injection of the knee? Scand J Rheumatol. 2012;41:6672.

16. Sibbitt WL Jr, Band PA, Kettwich LG, Chavez-Chiang NR, Delea SL, Bankhurst AD. A randomized controlled trial evaluating the cost-effectiveness of sonographic guidance for intra-articular injection of the osteoarthritic knee. J Clin Rheumatol. 2011;17:409-15.

17. Maricar N, Parkes MJ, Callaghan MJ, Felson DT, O’Neill TW. Where and how to inject the knee: a systematic review. Semin Arthritis Rheum. 2013;43:195-203. 